\title{
Dinámicas de la "cultura" Apuntes etnográficos entre mbya y jurua en la región de San Ignacio, Misiones, Argentina
}

\section{(4) Lucrecia Raquel Greco ${ }^{1,2}$ \\ 1 Programa de pos graduación en antropología Universidade Federal da Bahia. Salvador, Brasil 2 Universidad de Buenos Aires, Buenos Aires, Argentina \\ Correo electrónico: lucregre@yahoo.com.ar}

Recibido:

28 de enero de 2019 Aceptado:

13 de agosto de 2019

doi: 10.34096/runa.v40i2.5536

\section{Resumen}

Analizaré contextos donde diversos colectivos indígenas mbya y jurua (no indígenas) realizan acciones conjuntas que involucran nociones de "cultura" en la región de San Ignacio, Misiones, Argentina. Las reflexiones emergen de mi participación en un colectivo de trabajo audiovisual así como de mi investigación de posdoctorado en comunidades y una Escuela Intercultural Bilingüe aledañas a San Ignacio. Busco entender los modos en que se producen diferencias, identificaciones y alianzas entre los diversos colectivos mbya y jurua en los conflictivos contextos de cercamiento territorial contemporáneos. Contextualizaré estas interacciones en el marco de políticas de identidad estatales y profundizaré en los modos en que los colectivos mbya realizan proyectos de existencia al construir-resaltar su diferencia y relación ante la sociedad no indígena así como los modos en que los diversos colectivos jurua analizados acompañan estos proyectos.

\section{"Culture" dynamics: Ethnographic notes among jurua and mbyain San Ignacio area, Misiones, Argentina}

\begin{abstract}
I will analyze contexts in which mbya and jurua (non-indigenous) collectives lead up actions related to mbya "culture" in San Ignacio area, Misiones, Argentina. These considerations emerge from my participation in an audio visual working collective as from my post $\mathrm{PH}$ research between communities and in an Intercultural Bilingual School in San Ignacio area. I reach an understanding of the ways in which mbyaand jurua collectives produce differences, identifications and alliances in the conflictive contemporary contexts of territorial enclosure. I will contextualize these interactions in the frame of State identity policies and I will focus in the ways in which mbyacollectives perform their existence projects by building and by emphasizing their difference and
\end{abstract}

\section{Palabras clave}

Pueblo mbya; Relaciones Interétnicas; Políticas de Identidad; Cultura; Estado argentino

\section{Key words}

mbya; Interethnic Relations; Identity Policies; Culture; Argentine State 
relation in front of non-indigenous society. I will focus also in the ways in which the different jurua collectives support these projects.

\section{Dinâmicas da "cultura": notas etnográficas entre mbya e ju- ruá na região de San Ignacio, Misiones, Argentina}

\section{Resumo}

Analisarei contextos onde diversos coletivos indígenas mbya e jurua (não

Palavras-chave

mbya; Relações Interétnicas; Políticas da identidade; "Cultura"; Estado argentino
1. Indio es un término nativo, y el más usado en el Estado brasileño, para referir a los pueblos indígenas. indígenas) realizam ações conjuntas que envolvem noções de "cultura" na região de San Ignácio, Misiones, Argentina. As reflexões emergem da minha participação num coletivo de trabalho audiovisual assim como de minha pesquisa pós- doutoral em comunidades e numa Escola Intercultural Bilíngue lindeiras com San Ignácio. Procuro entender os modos em que são produzidas diferenças, identificações e alianças entre os diversos coletivos mbya e juruá nos conflituosos contextos de fechamento territorial contemporâneos. Contextualizarei estas interações no marco das políticas de identidade estatais e aprofundarei nos modos em que os coletivos mbya realizam seus projetos de existência construindo e destacando sua diferença e relação perante a sociedade não indígena, assim como os modos em que os diversos coletivos jurua analisados acompanham estes projetos.

\section{Introducción}

En el verano de 2012, Miguel Verá Mirim, entonces cacique de la aldea mbya Tekoa Mbo'yty, Camboinhas, Rio de Janeiro, Brasil, me explicaba, dialogando sobre un proyecto de video con la Universidad Federal Fluminense, que el hecho de encuadrarse a sí mismes como "indios" era una herramienta en la lucha por las tierras: “Nosotros somos indios, indios es un término de afuera, pero también es útil". Miguel también permitía y estimulaba la entrada de turistas a la aldea, hoy relocalizada en Maricá, para mostrarles algo de la "cultura" del pueblo mbya y hacer conocer su lucha por territorio. ${ }^{1}$

El "afuera", como el que reconoce Miguel, forma parte de las categorizaciones de la experiencia de diversas comunidades indígenas latinoamericanas. Joanne Rappaport, al reconocer entre les activistas indígenas del Cauca colombiano nociones de adentro y de afuera, propone pensar las mismas políticamente "como metáforas basadas en el espacio, que proyectan objetivos en el futuro y no tipologías culturales en el presente" (Rappaport, 2007, p.1). De este modo, usar, performar e incorporar categorías de "afuera" no implica minar formas de existencia propias sino que puede también ser un modo de generar herramientas para dialogar con ese "afuera" en el marco de proyectos políticos de autonomía (Rappaport, 2007).

Una de las categorías "de afuera" más presente en interlocuciones entre indígenas y no indígenas, y que estuvo presente a lo largo de la experiencia etnográfica que aquí citaré, es la de "cultura" (Wagner, 2010; Sahlins, 1997; Rappaport, 2007; Carneiro da Cunha, 2009). Esta categoría es fruto de dinámicas de opresión y lucha, herencias coloniales, ideologías multiculturales, políticas estatales y multinacionales de identidad-alteridad, emergencia de movimientos sociales 
entre otras (Wright, 1998; Abu Lughod, 2012; Lins Ribeiro, 2008; Sahlins 1997; Segato, 2007; Greco, 2016). En este complejo marco, Manuela Carneiro da Cunha (2009) distingue cultura y "cultura" con comillas, donde la primera refiere a esquemas interiorizados que organizan la percepción y acción de las personas y la segunda a los metadiscursos reflexivos sobre cultura. En este texto analizaré la categoría entrecomillada, considerando que si la "cultura" no es lo mismo que las categorías nativas para dar cuenta de la propia existencia ni englobaría todas las prácticas de los pueblos, podemos afirmar que emerge de dichas prácticas. Esta "cultura" se hace presente sobre todo en el contexto de interlocuciones con el "afuera" como procesos de escolarización, la recreación de performances, reivindicaciones ambientalistas, integración a sistema de salud estatales, proyectos de "desarrollo", entre otros. Así, utilizo "cultura" con comillas, desde una concepción no escencializante ni sustancialista, sino en cuanto categoría utilizada en los discursos de los colectivos. Las veces que el término aparece sin comillas está refiriendo a categorías nativas estatales, como políticas interculturales, entre otras. Para el caso mbya, los diversos modos de crear la "cultura" se realizarían desde el ñande reko, a su modo, desde su forma de ser (Meliá, 1981; Wilde, 2008), al producir de modo dinámico y situado, proyectos propios para crear su devenir como pueblo.

En este texto analizo algunas prácticas llevadas adelante entre diversos colectivos mbya y jurua (blanques) de la región de San Ignacio, Misiones, donde la "cultura" es un tema explícitamente abordado. Las reflexiones emergen de mi participación en un colectivo de trabajo audiovisual autogestionado, que se desarrolló entre los años 2008 y 2012 cuyo epicentro fue la aldea Katupyry (San Ignacio, Misiones) así como de mi investigación de posdoctorado realizada entre 2015 y 2016 en comunidades aledañas a San Ignacio y en la Escuela Intercultural Bilingüe de Katupyry junto con docentes mbya y jurua. Se trata así de una etnografía basada principalmente en la observación participante y en el registro de las discusiones colectivas realizadas con el equipo de trabajo audiovisual. Entiendo que el modo de construirse permanentemente como mbya y producir "cultura" se da en una coyuntura desigual, donde los colectivos indígenas realizan un trabajo intenso de identificación para llevar adelante su proyecto de autonomía ante la sociedad no indígena. Mientras tanto, los diversos colectivos jurua con quienes interactúan, llevan adelante procesos de reflexividad menos intensos o más lentos, en gran parte debido a su situación de "mayoría" - en el sentido de "constante de expresión o de contenido, una medida estándar" (Deleuze e Guattari, 1995, p. 52), y se identifican como plenamente, o al menos parte del Estados nacional (Estado que por su vez ha reconocido e integrado con más énfasis a los colectivos no indígenas a lo largo de su historia). ${ }^{2}$ En este trabajo me centro particularmente en el colectivo jurua docente y en el colectivo jurua de trabajo audiovisual que integré.

En la siguiente sección traeré algunas consideraciones sobre la historia reciente mbya en Argentina para entender cómo las relaciones interétnicas se han constituido en el marco de tensiones entre las históricas políticas homogeneizantes estatales argentinas, la interferencia de más recientes políticas de la diferencia y las políticas mbya. ${ }^{3}$ Respecto al papel del Estado, entiendo que algunos aspectos de la experiencia nacional forman parte de las prácticas de les "otres" de los Estados nacionales, como los pueblos indígenas (Briones, 1998; Segato, 2007). Estos aspectos son principalmente la reterritorialización, la inserción en instituciones escolares, de salud, fuerzas armadas, trabajo, el contacto con medios masivos de comunicación y el mercado (Yúdice, 2002).
2. Diverses autores han apuntado el modo en que el Estado Argentino, como la mayoría de los Estados latinoamericanos, tendió a "alterizar" a las poblaciones indígenas (Briones, 2005; Segato, 2007). Carla Golé y María Lucila Rodríguez Celín (2017) reconocen para la región los modos en que el Estado provincial y nacional integró a colones y criolles en sus políticas de identidad antes que a los colectivos indígenas.

3. Refiero a una noción de relaciones interétnicas procesual que no define a priori los grupos sino que piensa cómo estos se constituyen en el marco de relaciones de poder (Cardoso de Oliveira, 2003). 
4. Durante esta investigación también establecí algunos diálogos con la citada aldea Tekoa Mbo'yty, Brasil, experiencia que también informa parcialmente mi visión sobre las maneras situadas de los colectivos mbyade interactuar con los "afueras".
En la segunda sección trabajaré sobre mi experiencia etnográfica para entender diversos modos en que se producen diferencias, identificaciones y relaciones entre diversos colectivos mbya y jurua en los contextos de cercamiento territorial y existencial contemporáneos.

\section{Dinámicas mbya en el Estado}

Ticio Escobar (2015) caracteriza a les mbya que viven en Paraguay como uno de los pueblos indígenas que tiende a ser más "conservador", al distinguir "con cuidado lo propio de lo ajeno" y al problematizar "la necesidad de absorber o rechazar los signos extranjeros" (p. 35-36). Tal descripción, aunque un tanto generalista, puede aplicarse a las situaciones analizadas en este texto, donde les interlocutores mbya tienden a resaltar lo propio en los discursos sobre la "cultura" ante otros actores no mbya, así como a pensar y discutir colectivamente las diversas estrategias de relación a ser adoptadas con estos colectivos. A tono con esta frase de Escobar, basada en comparaciones con otros pueblos indígenas que habitan el actual Paraguay, es importante aclarar que este texto emerge de mi investigación posdoctoral, donde analicé comparativamente las posiciones de colectivos qom del Oeste de Formosa y mbya residentes en la región de San Ignacio en el marco de acciones enfocadas en la "cultura" junto con colectivos no indígenas. ${ }^{4}$ A partir de mis experiencias en campo, observé que mientras les mbya se preocupaban por resaltar su especificidad y diferencia en el presente, les qom acentuaban que la "cultura" propiamente indígena se encontraba sobre todo entre les "antigües", quienes no pasaron por procesos de cristianización y tuvieron menor contacto con los Estados nacionales (Greco, 2016; Gómez et al., 2013). Esta diferencia entre qom y mbya se entiende tanto a partir de los proyectos de autonomía y resistencia específicas de los colectivos como de los diferentes procesos de ocupación del territorio indígena por parte del Estado en el siglo XIX y comienzos del XX, considerando especialmente las formaciones regionales de alteridad fruto de las fronteras administrativas impuestas por el Estado (Briones, 2005). Así, en Misiones, la presencia del Estado fue diferente a aquella del Chaco o de la Patagonia, donde la estrategia fue el genocidio a través de la invasión militar. En la actual Misiones no hubo campañas militares sistemáticas en el período de expansión del Estado nacional. Asimismo, hasta comienzos del siglo XX la negación oficial de la presencia indígena y la evitación del contacto por parte de les mbya fueron circunstancias que contribuyeron a reforzar su autonomía (Enriz, 2010; Cebolla Badie y Gallero, 2012). Otro hecho relevante que propiciaría la marcación constante del espacio de lo "propio" fue que les mbya tuvieron en los últimos dos siglos mayor contacto con criolles y colones y menos con misioneres centrades en catequizar y modificar sus modos de vida. Asimismo, en la región de Misiones, lxs mbya no trabajaron masivamente para 1xs colonxs (Cebolla Badie y Gallero, 2012), mientras los vecinos pueblos del Chaco fueron forzados a integrarse al trabajo en los ingenios azucareros desde comienzos del siglo XX (Gordillo, 1996). Noelia Enriz, (2011) apunta también que la autonomía mbya respecto de les colones y del Estado pudo deberse a la ausencia de vínculos comerciales, la subsistencia con recursos del monte y al propio "modo de vida mbya", el cual, destacamos, es dinámico y situado y se recrea en contextos socio-ecológico-territoriales específicos (Barbosa y Mura, 2018).

Para procesos más antiguos, debemos considerar que - aunque la historia de la misionalización y la pos misionalización guaraní de la región de las misiones presenta diversas facetas en las relaciones entre indígenas y no indígenas, 
pasando por resistencias, negociaciones, mestizajes y antagonismos (Wilde, 2001; Gorosito Kramer, 2006), les mbya pasaron a mantener contacto más intenso y continuo con los no- indígenas a partir de la expansión de las fronteras agrícolas internas de los Estados nacionales de Argentina, Brasil y Paraguay, entre fines del siglo XIX y comienzos del XX, período durante el cual también buscaron limitar los contactos retrayendo su territorialidad, aunque también se integraron al circuito agrícola productivo como trabajadores asalariados en distintas formas (Gorosito Kramer, 1982; Garlet, 1997; Assis y Garlet, 2004; Bartolomé, 2009).

En lo que refiere a la historia reciente, a pesar de las citadas relaciones y contactos, hasta la década de 1950, les indígenas fueron invisibilizades en documentos y medios de comunicación de Misiones. En 1966, se realizó un censo para dar cuenta de las poblaciones indígenas, ${ }^{5}$ aunque hasta mediados de la década de 1980 se hablaba en los medios de su inevitable extinción. Las décadas de 1970 y 1980 se caracterizaron en la provincia de Misiones por el violento cercamiento del territorio mbya que se dio sobre todo con la reconversión productiva hacia la explotación forestal y la concentración de la propiedad de la tierra, afectando la posibilidad de mantener prácticas ligadas al uso del monte, constitutivas de las reivindicaciones del modo de ser mbya. El Pueblo mbya se auto identifica como habitante de las selvas nativas (Chase-Sardi, 1989; Wilde, 2008), siendo la aldea y el "monte" (tal como se lo llama en la región) ámbitos complementarios, donde el último es proveedor de alimentos y fundamento del modo de ser mbyá (Meliá, 1986). En este sentido, el cercamiento territorial afectó tanto los modos de ser con el monte como las formas de ejercer la movilidad territorial, también constitutivas del ñande reko (Cadogan, 1960; Clastres, 1993; Bartolomé. 2009). En este contexto de cambios aparecen demandas mbya frente al Estado, especialmente en lo que refiere al pedido de escuelas, títulos de propiedad y documentos de identidad (Bartolomé, 2007; Enriz, 2010, 2011; Golé y Rodríguez Celín, 2017).

Precisamente en este período, en el que las comunidades perdían territorio y debían modificar más abruptamente sus condiciones de existencia en relación con la selva paranaense, los organismos del Estado debieron admitir la presencia indígena y, en alianza con la iglesia católica, basaron sus acciones con la población mbya en la creencia de extinción inevitable, buscando convertir las a comunidades en grupos productivos en sus tierras o en fuerza de trabajo. El Proyecto de Desarrollo Integral de las Comunidades Guaraníes de Fracrán y Peruti creó las primeras escuelas católicas dentro de las comunidades (Enriz, 2010). Estas escuelas se instalaron sin gradualidad: de no frecuentar ninguna institución les niñes pasaban a ir a escuelas doble turno en castellano y sin un programa intercultural pensado en conjunto (Quadrelli 1997, en Cebolla Badie, 2005). Ya desde estas primeras articulaciones algunes mbya pidieron al obispado que se respetara su modo de ser enfatizando su voluntad de preservar su autonomía (Enriz, 2010).

Ante la avanzada de los movimientos indígenas y tras el fracaso de los proyectos pensados sin su participación, se fueron delineando políticas estatales más inclusivas con los pueblos originarios. En 1987 se sancionó la Ley № 2.435 que reconocía los sistemas políticos, sociales, económicos y culturales guaraníes en Misiones, ley que fue substituida por la creación de la Dirección Provincial de Asuntos Guaraníes (DPAG) a partir de la Ley № 2.727 de 1989 (Enriz, 2011). Esta entidad ha sido dirigida por juruas, ha mantenido discursos paternalistas y su representatividad ha sido constantemente cuestionada por diversos colectivos mbya. Desde la década de 1990 se comenzaron a reformular
5. Se trata del Censo Nacional Indígena, que dio como resultado 1.500 habitantes, generando repercusiones en los medios gráficos locales (Enriz, 2011). 
6. Marilyn Cebolla Badie (2005) comenta que en 2005, en reuniones para pensar las escuelas, les maestres mbya resaltaban que "no necesitaban que nadie les rescatara su cultura porque el mbya reko está vivo" y que sus necesidades se centraban en conocer mejor el mundo

del blanco. Les mbyahablando con les jurua de "cultura" traen la noción reko, frecuentemente traducida como costumbre o modo de vida (Cretton, 2010) y la marcación de autonomía y diferencia respecto al mundo no mbya. Diverses interlocutores en campo, en el año 2016 continuaban expresando que la escuela tenía sentido como espacio para aprender del mundo jurua y lidiar con él, y no para aprender el modo de vida mbya. Es interesante señalar diferencias con el mundo qom que conocí, donde en ciertas coyunturas algunas instancias del espacio escolar eras consideradas como una posibilidad de conversar sobre historia y cultura "aborigen".

7. Vale resaltar la asimetría: la participación indígena es como auxiliares y no como titulares.

8. Se trata de una proliferación de escuelas primarias. Las escuelas secundarias son minoritarias en el contexto de

la EIB Argentina en general.

9. Ana María Gorosito Kramer (2006) apunta que los liderazgos políticos guaraníes han sido dinámicos y dependientes de la conducta del líder. Destaca que aunque los Estados Nacionales han tendido a trabar estos procesos, las comunidades persisten en la búsqueda de estructurar de esta forma sus organizaciones políticas.

10. Entendemos por proyectos "una combinación de emprendimientos culturales, políticos y económicos [que dependen] de agentes externos tanto como de la población indígena- y que siempre son simultáneamente políticos, culturales y económicos" (Carneiro da Cunha, 2009, p. 340).

11. A lo largo de mi investigación las comunidades han desarroIlado proyectos de apicultura, plantíos de árboles y huerta, y los líderes se han manifestado preocupados por la continuidad de dichos proyectos, preocupación que pueden pensarse tanto como una inquietud relacionada a la subsistencia pero también a estimular relaciones con el uso del monte y la actividad agrícola, constitutivas del devenir mbya. los proyectos educativos, al introducir las perspectivas de la interculturalidad, lo que supondría pensar en el desarrollo de políticas educativas las relaciones entre diversos grupos sociales, entendiendo también las asimetrías que constituyen dichas relaciones (Dietz, 2017). En esa década los movimientos indígenas a nivel mundial junto con organizaciones internacionales lograron promulgar reformas constitucionales que favorecieron los derechos educativos indígenas. En Argentina se concretó entonces la Reforma Constitucional de 1994 donde se reconoció la pre-existencia de los pueblos originarios y se garantizó un marco general para la protección de sus idiomas y sus derechos educativos. Comenzó entonces un proceso de abandono de algunos lineamientos asimilacionistas para enfocarse, al menos en la letra de la ley, en el reconocimiento de diferencias "culturales". En este proceso, no obstante, se continuó marcando la posición de alteridad de les indígenas frente al Estado. Asimismo, y como en otros Estados latinoamericanos, el reconocimiento legal no bastó para propiciar de modo efectivo condiciones materiales para la reproducción de modos de vida autónomos de los pueblos originarios. Briones (1998a) apunta que las paradójicas dinámicas de reconocimiento de la presencia de pueblos nativos en cuanto "distintos" y la postulación de la necesidad de incorporarlos por parte de los poderes hegemónicos, así como las dinámicas de resistencia de los pueblos - utilizando muchas veces los recursos hegemónicos para mantener su autonomía - han sido y son procesos estables en América Latina. Así, si bien el discurso de la diversidad ha cambiado, las dinámicas de poder y resistencia persisten, como podemos notar en los procesos aquí descritos.

En la década del 2000, les mbya, comenzaron a solicitar con más énfasis la instalación de escuelas en sus comunidades, al pensarlas como espacios para aprender a leer y escribir en castellano (Cebolla Badie, 2005). ${ }^{6}$ La posibilidad de participación de adultes mbya como ADI (auxiliar docente indígena) ${ }^{7}$ y la práctica de coplanificación fueron aumentando el interés mbya en la escolarización (Bañay, 2015). ${ }^{8}$ En este último periodo también se extendió entre las comunidades el uso del castellano y aumentó la presencia de las ONG. La DPAG impuso una dinámica de asentamientos en aldeas según la cual cada una debería contar con un/a líder/eza política (cacique) y un líder espiritual (opygua, que lleva adelante las ceremonias e interviene en procesos de cura). Así se separaron y cristalizaron ambos papeles ${ }^{9}$ y se promovieron lógicas territoriales y administrativas exógenas, dado que hasta entonces, en la historia reciente no necesariamente cada núcleo poblacional contaba con ellos (Marilyn Cebolla Badie, comunicación personal, diciembre de 2016). En este proceso la mayoría de las aldeas pudieron mantener líderes político-administrativos pero muchas no lograron sostener un líder espiritual. En este sentido diversos caciques con los que conversé expresaron su preocupación por el hecho de que en los últimos tiempos cada vez menos aldeas cuentan con un opygua y en muchos casos ni siquiera con un opy (casa de reza) y han manifestado su interés por tener uno en cada aldea, como un modo de fortalecimiento comunitario. Asimismo, algo que fue impuesto por el Estado en un momento (la intención de que exista un líder espiritual en cada aldea) pasó a ser un elemento clave en las comunidades actuales en su lucha por mantener la autonomía y en el proceso de continuar siendo comunidad mbya.

También especialmente desde la década de 2000, las comunidades mbya fueron foco de diversos "proyectos" 10 de desarrollo, productivos, de asistencia, entre otros, llevados adelante desde organismos multilaterales y ONG. ${ }^{11}$ Asimismo, se han ido creando cada vez más grupos de performance, especialmente coros, que se presentan ante públicos no mbya y mbya. En su análisis de casos en Brasil, Valeria Macedo (2013) apunta que a través de los coros y otras 
manifestaciones, se enuncia la singularidad guaraní ante el público no indígena, lo que contrasta con la "invisibilidad cultural" previa y trastoca el sentido de la diferencia, que de ser fuente de humillaciones y conflictos interétnicos pasó a ser un modo de imponer respeto. Este movimiento de reivindicación identitario ante otres a través los coros y otras performances puede ser identificado en el caso de diversos colectivos mbya de Argentina, tal como en el del coro de la Escuela que aquí analizaré. Estas performances también se dan en contextos que presentan mayores tensiones entre dinámicas de resistencia y explotación, tales como la industria turística (Cantore y Boffelli, 2017) y la del espectáculo, donde, como observaremos, se presentan conflictos con algunos productores artísticos-mediadores. ${ }^{12}$ Como comentaré más adelante, en los casos de las performances artísticas algunas comunidades cuestionan el rol de les mediadores y buscan formarse para la gestión de los proyectos en todos sus aspectos. En definitiva, todos los procesos interétnicos poseen bemoles que deben ser entendidos en las coyunturas específicas y teniendo en cuenta las posiciones de poder y los posicionamientos de los colectivos. En la próxima sección discutiré estos temas a partir de la etnografía.

\section{Dinámicas de la "cultura"}

Elizabeth Pissolato (2012) señala que el "código de la 'cultura"' entre algunos colectivos mbya que viven en Brasil, se intensificó en las interlocuciones con jurua especialmente para la formulación de "proyectos". En la región de Argentina donde trabajé se da un fenómeno similar en el marco de las relaciones interétnicas. Iniciaré aquí entonces un recorrido etnográfico en el que indago las posiciones de distintos colectivos mbya y jurua en situaciones donde se pone en acción dicho código. ${ }^{13}$

Katupyry se encuentra a doce kilómetros de la ciudad de San Ignacio, es la comunidad mbya de mayor extensión del municipio homónimo (400 ha) y una de las pocas que cuenta con título legal de sus tierras, situación que posibilita una mayor dedicación a actividades de subsistencia y permite mantener una relación más intensa con el monte en relación con otras aldeas de la región (Padawer et al., 2013). La Escuela Intercultural Bilingüe de Katupyry, creada en 1995, dependió hasta mediados del 2013 del Obispado de Posadas, y fue una escuela católica de gestión privada funcionando formalmente con la modalidad de Educación Intercultural Bilingüe (EIB) en la provincia desde 2006, momento en que se incorporaron ADI de la comunidad. Sin embargo, ante sucesivas protestas realizadas por la comunidad debido a la falta de diálogo con las autoridades religiosas, la directora fue desafectada del cargo, que fue asumido por la docente jurua de mayor antigüedad. Tras la expulsión de las autoridades religiosas, les docentes mbya conquistaron mayor espacio y libertad para su trabajo y la escuela pasó a tener mayor aceptación entre la comunidad. Les docentes mbya se encuentran satisfechos con la administración actual de la institución por propiciar mayor libertad y autonomía, pero también sostienen que el interés por tener una escuela tiene como objetivo adquirir herramientas del "blanco". Padawer et al (2013) destacan el trabajo de esta escuela con relación a otras de la región, especialmente por el lugar central de la pareja pedagógica jurua-mbyay la importancia dada al espacio curricular "Lengua y Cultura Mbya", que contrastan con las tendencias castellanizantes y dan mayor protagonismo al docente mbya. Asimismo, parte de las docentes jurua, generalmente criollas locales (hay solo un hombre en el plantel no indígena), se empeñan en aprender la lengua, conocer prácticas de la comunidad y en realizar formaciones en bilingüismo, así como en acompañar a la
12. Alfonsina Cantore y Clara BoffeIli (2017) analizan el trabajo mbya con el turismo en Iguazú. Entienden que, si bien las presentaciones son fuente de reivindicación simbólica y estrategia de subsistencia económica, esta se sitúa a precio de reproducir relaciones de dependencia vinculadas a la expropiación del territorio y la dependencia del mercado.

13. Diverses interlocutores mbya en Argentina (caciques, líderes, estudiantes), decían en el año 2016 que percibían que quienes vivían en Brasil tenían mayor manejo de la gestión de proyectos culturales, como festivales, materiales de divulgación y procesos de investigación. 
14. Entre les jurua la docencia es un rol principalmente femenino mientras entre les mbya estos papeles, ligados a dinámicas interétnicas, han sido mayoritariamente masculinos. Estos roles son objeto de reformulaciones ante el fortalecimiento de grupos de mujeres mbya.

15. Esta inserción de colones, ligada generalmente a la falta de establecimientos educativos en las colonias, simetrizaría las políticas de identidad: en estas situaciones son colectivos no indígenas quienes toman horas de "cultura" mbya. comunidad en sus demandas. Todes elles, así como les docentes mbya permanecieron en la Escuela tras la ruptura con la administración anterior, pues decidieron apoyar el reclamo de la comunidad. Les docentes mbya son en su mayoría hombres, aunque desde 2016 el equipo docente se está proponiendo explícitamente la inclusión de más mujeres en el plantel. ${ }^{14}$ Asimismo, cabe mencionar que en el período en que realicé trabajo de campo, se encontraban matriculades algunes niñes criolles, situación que se da en otras EIB de la zona (Golé y Rodríguez Celín, 2017). ${ }^{15}$

La escuela de Katupyry, como todas las EIB de la región, es un espacio en constante negociación configurado por nociones de persona y saber jurua: se presupone que el conocimiento se crea entre humanes, en una sala, sentades, a través de la palabra escrita, prácticas que contrastan con modos de conocer mbya, los cuales son desarrollados través de vínculos con seres humanes y no humanes y a través de la práctica (Cabral y Keese, 2014.) Desde diversas negociaciones y límites, en el cotidiano de la Escuela, docentes mbyay jurua construyen un proyecto conjunto definiendo adentros y afueras. Relataré aquí algunas escenas que dan cuenta de esta construcción conjunta mbya-jurua.

En una jornada de junio de 2016 se preparaba una visita de una escuela primaria privada de clase media alta de Buenos Aires. Ese día las maestras jurua hicieron doble turno, intensificaron la limpieza y cocinaron tortas fritas, junto con algunas mujeres de la comunidad. Al llegar, las visitas entregaron donaciones de ropas y bolsas de golosinas y posteriormente el coro de niñes de la Escuela se presentó. Animades por un profesor de la escuela visitante, les niñes jugaron al futbol y otros juegos. Finalmente, les adultes comenzamos a repartir la merienda, priorizando a les niños invitades, quienes la tomaron y la repartieron entre les niñes mbya, que la aceptaron. Como observadora externa, proveniente de Buenos Aires, puedo suponer que les invitades no quisieron tomar la merienda por nociones prejuiciosas de higiene o salud acotadas por experiencias urbanas de algunos colectivos clase media alta (falta de conocimiento sobre salud e higiene en contextos rurales, por ejemplo) y/o por su percepción de estar en una comunidad carente, lo que les llevaría a evitar comer la comida de quienes tendrían menos recursos económicos que ellos. Aunque el porqué de esta actitud es una incógnita, el hecho es que la merienda compartida acabó siendo consumida solo por les niñes locales. Al terminar el acto y al día siguiente, les profesores mbya y jurua de la escuela reprendieron a les niñes mbyapor haberse comido todas las tortas fritas. El argumento fue que las visitas habían venido a entregar donaciones y que, además de un acto de agradecimiento, hubiera sido una novedad para elles merendar tortas fritas y mate cocido.

En la escena narrada les docentes jurua expresaron una intensa preocupación por recibir bien a las visitas, en función de lo cual trabajaron doble turno, cocinaron y movilizaron a sectores de la comunidad para esta recepción. Por su parte, les docentes mbya muestran, selectivamente y de un modo previamente acordado con les jurua locales, algo de su "cultura", a través del coro y las artesanías a les jurua de Buenos Aires. Les mbya junto con les maestres jurua locales eligen dar a les jurua visitantes artesanías hechas por ellos con materias primas del monte, mientras que les jurua foráneos dan a les mbya productos industrializados e impersonales: ropas usadas y golosinas, objetos que no necesariamente son leídos como de su "cultura". La elección de mostrar algo de la propia "cultura" a espectadores externos es, por un lado, emergente de la posición de minoría en la que son colocades les mbya y los pueblos originarios en general, pero es también una estrategia mbya para representarse y realizar 
su proyecto de vida en situaciones de contacto, mostrando sus saberes y la materialidad de los mismos en prácticas concretas. En lo que refiere al reclamo por las tortas fritas, docentes locales, mbya y jurua naturalizaban ante les niñes mbya algunos papeles de dadores y receptores, sin cuestionar las relaciones de poder y asimetrías que se encuentran implícitas en las donaciones. Sin embargo, fuera de estas interlocuciones con les niñes mbya, tanto les docentes jurua como los mbya suelen cuestionar los diversos modos y contenidos de las donaciones, entendiendo que no todas son pertinentes y ejerciendo una mirada crítica sobre quienes se acercan a donar sin conocer antes las reales necesidades. Por ejemplo, comentaban que suelen llegar remedios para problemas de salud como diabetes, ausentes en las comunidades, o vestimentas que les mbya no usan, como ropas de lana y abrigo que llegan en verano y no tienen lugar donde ser guardados, talles demasiado grandes, número de calzado demasiado altos, corbatas, entre otras. No obstante, tanto docentes jurua como mbya prefieren recibir las donaciones, generalmente consistentes en ropa, comida y medicamentos- como una forma de suplir carencias ante una realidad donde la situación de alimentación (con el mencionado cercamiento territorial y la progresiva dependencia del Estado y el mercado), salud (con aumento de enfermedades y mayor dependencia del sistema médico estatal) y acceso a servicios y productos son críticas en las comunidades.

Así como les mbya reconocen su "cultura" para poder presentarse ante otres, les maestres jurua locales no articulan un discurso que objetive sus propias prácticas ni las de otres jurua. Generalmente, los discursos entre les maestres jurua tienden a resaltar lo "curioso", "extraño" o "cultural" de las prácticas que les resultan diferentes. Las prácticas escolares, el saludo a la bandera, la formación de filas, entre otras, no son cuestionadas ni por les jurua ni, al menos explícitamente, por les mbya. En algunas ocasiones les docentes jurua valoran positivamente saberes mbya, especialmente en lo que refiere a conocimientos del monte, las medicinas y las artesanías, pero se muestran a menudo sorprendidos o indignados con prácticas de parentesco o religiosas. Sin embargo, como señalé anteriormente, a pesar de la presencia de ciertos paradigmas etnocéntricos, en la escuela se realiza un trabajo lento de descentramiento acompañando a las comunidades en sus demandas y buscando profundizar en el conocimiento de les mbya. Así, les docentes no refuerzan estigmas como en otras escuelas analizadas en Misiones, donde existe una explícita condena por parte del plantel jurua sobre varias prácticas mbya (como por ejemplo en los casos retratados por Cebolla Badie, 2005). Les docentes jurua saben que acompañan un proyecto mbya, especialmente desde la expulsión de las autoridades religiosas. Elles se preocupan por visibilizar y valorizar positivamente a los mbya y su "cultura" ante el resto de los jurua, a través de la búsqueda por ocupar espacios públicos y por difundir el trabajo en el pueblo de San Ignacio. Así, cuando existen invitaciones de la intendencia para festejos públicos, les docentes intentan que les niñes puedan presentarse. La directora de la Escuela señala que la intendencia no suele apoyar cuidadosamente la presencia mbya en los actos, lo que se demuestra en la negligencia en relación con los cuidados con el transporte, la alimentación, entre otras cosas. No obstante, les docentes prefieren que les niñes estén presentes para poder dar visibilidad al trabajo de la Escuela y a las comunidades mbya.

Una situación que evidencia una mayor dificultad de reflexividad jurua en cuanto "mayoría" es la gestión de la merienda escolar. En 2016 los recreos de la escuela, se instalaba en la cantina una mesa de golosinas industriales que se vendían para juntar fondos para la escuela, mientras una anciana de la comunidad preparaba el maíz pisado con azúcar, que se daba y no se vendía. 
Así se ofrecían dos opciones de merienda escolar, la segunda de las cuales era una propuesta de la comunidad, que la gestión religiosa anterior había negado. Es interesante considerar algunas perspectivas de les docentes jurua sobre el consumo de azúcar. Por un lado les parecía normal que les niñes mbya consumieran grandes cantidades de azúcar en formato de golosinas industriales. Sin embargo cuestionaban como extrañas las prohibiciones que pesan sobre el consumo de azúcar durante la menstruación -prohibición ligada al ciclo de la vida de las mujeres, inherente a modos mbya locales de constituirse como persona- considerándolo un hecho extraño y represivo, parte de la "cultura". Mientras tanto, cuando yo les conté que no quería que mi hija de un año y medio, allí presente, consumiera golosinas con azúcar, entendieron y estuvimos conversando largamente sobre los efectos contraproducentes de la sustancia para la salud. Así, se nota una asimetría donde mi posición es validada y las elecciones de les mbya son "culturizadas". Del mismo modo se expresa un desconocimiento por parte de les docentes jurua de la importancia de la alimentación en los modos mbyade constituirse como persona (Macedo, 2013). Otro desentendimiento que lxs docentes jurua relataron fue que demoraron años en comprender por qué los padres de niñes recién nacides no se alejaban de la aldea, hasta que recientemente les adultes les explicaron el sentido del periodo de couvade (prácticas de paternidad en relación a les recién nacidos), que entre les mbya consisten en la necesidad de la proximidad física de padres e hijes: en caso de que los primeros se alejen mucho o se expongan a riesgos, peligra la vida de les niñes (Cebolla Badie, 2017). Estas prácticas se relacionan con un modo de existencia mbya de acuerdo con el cual la potencia de los cuerpos aumenta o disminuye en relación a las afecciones con seres humanes y no humanes (Macedo, 2013). Les docentes, desde una noción antropocéntrica e individualista del cuerpo como entidad autocontenida, interpretan esta y otras prácticas como un problema de represión o creencia "cultural". Este desentendimiento es dado en gran parte por estructuras etnocéntricas que impiden la comprensión de diversos modos de existencia y de constitución de persona y más específicamente por la falta de formación política-pedagógica. Otro tipo de formación llevaría incluso a cuestionar la definición de "cultura" que aquí analizamos, entendiendo los contextos políticos donde fue gestada, así como su matriz antropocéntrica.

En lo que refiere a los desentendimientos mencionados les docentes comentan que fue muy difícil que les mbya respondieran preguntas sobre el sentido de sus prácticas, cosa que hicieron muchos años después de trabajar juntes. Les docentes también tomaron conocimiento de esos sentidos a través de otros medios, como cursos de formación en EIB o lecturas. El problema de la falta de respuesta de les mbya puede pensarse como un modo mbya de sostener algunos métodos de aprendizaje propios, no basados en preguntas y explicaciones, sino en la experiencia práctica y la observación (Cabral y Keese, 2014). Así, estos secretos se constituyen en una modalidad de mantener lo propio, donde quienes acceden al conocimiento desde "afuera" lo hacen una vez que aprenden las lógicas prácticas de aprendizaje mbya. Esto no siempre se da así, sino que depende de les actores involucrados, de los contextos y elecciones, como señalaré en otros ejemplos donde el refuerzo de lo propio se da mostrando, y explicando.

A diferencia de les docentes, que quieren dar a conocer a los mbya sin ningún interés de lucro, algunes "gestores culturales" jurua entienden la "cultura" como un valor agregado que pueden usar para su beneficio. En este sentido, el cacique de otra comunidad aledaña a San Ignacio, me manifestó su 
inconformidad con la gestión de la venta y distribución de discos del coro de su comunidad hecha por un músico jurua. El cacique, que trabajó en DPAG y en otros espacios con jurua, también relató que existe una tendencia a no pagar las presentaciones de les mbya en shows artísticos, por lo cual comenzó a exigir este pago. El cacique tampoco aprueba que les jurua canten canciones mbya porque considera que en este acto les mbya son desvalorizados como intérpretes. Así, en su tránsito por instituciones jurua y sus negociaciones con artistas jurua el cacique fue consolidando una posición de defensa de la autoría mbya frente al mundo no mbya así como una posición crítica de la mediación jurua sobre procesos de representación mbya.

El colectivo de educación y producción audiovisual del que participé, también se cuestionó cómo, para qué y para quienes representar la "cultura" mbya. El grupo, compuesto por educadores visuales y científiques sociales entonces residentes en Buenos Aires, se formó cuando una de sus integrantes, que acompañaba a las comunidades desde el 2001, percibió la necesidad de apoyo en la documentación y definición de líneas de acción ante la búsqueda mbyade aprovechar el espacio escolar como herramienta. La comunidad nos llamó a colaborar, a través del vínculo estrecho de confianza con nuestra compañera, pues la escuela, en ese momento, estaba siendo gestionada por las autoridades religiosas siendo un espacio hostil que no les daba margen de acción y decisión. La intención de nuestro colectivo fue generar estrategias para no representar a lxs mbya, permitir la emergencia de narrativas propias e interferir lo menos posible en el devenir cotidiano de la aldea. También tuvimos como acuerdo evitar miradas académicas que en ese momento considerábamos asimétricas. Todas estas intenciones tuvieron, como señalaré, implicancias en la práctica.

En el primer viaje a Katupyry en 2008, nos reunimos con referentes de la comunidad, que decidieron que nuestra colaboración se enfocaría en proporcionar herramientas para registrar la vida cotidiana y generar material didáctico en lengua mbya para las escuelas como recurso para la preservación y el registro de diversos aspectos emblemáticos del ñande reko. Se manifestaba el interés de complementar con soportes audiovisuales la transmisión intergeneracional mbya y una sensación de estar atravesando un proceso de pérdida.

En enero de 2009, acordamos realizar talleres en Escuela Familia Agrícola Guaraní (EFAG) Tajy Poty, en la aldea Yacutinga. La EFAG era entonces una de las referencias más importantes de búsqueda intercultural donde cursaban la secundaria muches alumnes que actuaban como ADI. En asambleas realizadas en Katupyry y posteriormente en las aulas, se decidió abordar temas como espiritualidad, educación no escolar, el pasado y el abandono de ciertas prácticas comunitarias, como los saludos a les ancianes, la construcción de opy, entre otras. En agosto de 2009, parte de nuestro equipo realizó los talleres en la EFAG utilizando horas aula. La propuesta educativa se centraba en buscar espacios y tiempos de trabajo que las comunidades dispusieran, asumiendo características diferentes a las de los procesos escolares. Los rodajes se llevaron a cabo en Katupyry, donde se continuó la formación técnica en el manejo de cámara en reuniones al aire libre y charlas, buscando integrar la formación a prácticas de comunicación y espacios cotidianos (fogones, arroyos, salida al monte, etc.). Las metodologías utilizadas apuntaban al desarrollo de estéticas y narrativas propias mbya. Así, en todos los casos les estudiantes y referentes de la comunidad filmaron y desempeñaron los roles técnicos mientras nuestro equipo asistía y asesoraba. Se realizaron rodajes con el opygua, y otros ancianos que contaron a los jóvenes, en mbya, cómo se organizaban las actividades de 
16. Esto contrastaba con otras experiencias enfocadas en públicos externos, como las de comunidades de Brasil que venían filmando con apoyo jurua desde comienzos de 2000 con traducciones al portugués (Macedo, 2013). la comunidad en el pasado. La elección de realizar registros en lengua mbyase vinculaba a la búsqueda de priorizar la comunicación intra mbya y a que nuestro rol fuera de asesores técnicos sin entrometernos en las decisiones de la comunidad. ${ }^{16}$ Aunque, como en muchos otros casos de trabajo enfocados en la "cultura" mbya con colectivos de "afuera", fueran les jóvenes y adultes quienes se comprometían con el proyecto, la consulta con les ancianes y el foco en sus saberes fue central en el proceso. En 2009, un ADI nos llevó a una comunidad formada solo por una familia donde su padre era cacique. El anciano recibió nuestra visita, nos contó y mostró muchas cosas, decidió filmar en castellano y nos invitó a una ceremonia dentro del opy. Pueden notarse diversas estrategias de sostener la autonomía según la posición de las comunidades: existen líderes que prefieren mostrar y otres que prefieren ocultar, así no siempre y no ante todos los colectivos el secreto es la estrategia de autonomía. En este caso para el anciano, la divulgación hacia afuera, filmar en castellano ante público jurua e invitarnos al opy reforzaba la lucha por el territorio en el acto de la documentación y posible divulgación. Hacerse conocer era, como él mismo resaltó, una herramienta para valorizarse y defenderse.

La edición de los materiales generados por nuestro equipo (registros de la vida cotidiana, producción de artesanías, reuniones y conversaciones, ejecución de danzas y cantos tradicionales y del proceso de filmación) comenzó en la comunidad y continúo en encuentros en Buenos Aires y San Ignacio, siempre procurando dejar copias e insumos tecnológicos en las comunidades.

El equipo audiovisual jurua funcionó en forma difusa, con acciones esporádicas hasta el año 2015, siendo una de las últimas acciones la colaboración en tomas de video para un diccionario audiovisual mbya internacional. En 2016 decidimos que no podíamos más reconocernos como colectivo y nuestra relación con las comunidades pasó a ser individualizada.

A lo largo del proceso, e incluso en el año 2016 cuando retorné a Katupyry, les jóvenes y algunes adultes y ancianes resaltaron que las actividades realizadas se habían constituido en un espacio de encuentro intergeneracional que estaba descuidado y enfatizaban la importancia de estar trabajando de esta manera con la "cultura" o con preguntas sobre cómo sostener "ñande reko".

En lo que refiere al funcionamiento de nuestro grupo, la reflexividad, en cuanto pensamiento crítico sobre nuestras posiciones y las de nuestres interlocutores, fue un componente permanente. Nuestra posición generó este interesante vínculo con las comunidades, pero también limitó algunas formas de los diálogos en territorio, pues, por ejemplo, la búsqueda de despojarnos de algunas partes de nuestra subjetividad académica, eliminándola, en lugar de problematizarla abiertamente no permitía la emergencia de una reflexividad más agudizada sobre el lugar que efectivamente estábamos ocupando. La evitación de la mirada académica se basaba en una concepción de antropología y quehacer de las ciencias sociales específica, donde les sujetes serían tratades como objetos, situación que no refleja gran parte de las prácticas académicas de investigación contemporáneas entre pueblos indígenas (ver Albert, 1997) ni nuestras propias posiciones como profesionales, sino que consistía en un cierto prejuicio y una necesidad de separación de esferas de acción propias de nuestro colectivo. También me interesa apuntar que el modo en que ejercimos nuestra reflexividad era informado, paradójicamente, tanto por nuestra formación académica como por nuestras trayectorias de vida y también por el hecho de que el trabajo en la aldea no era ni nuestra fuente de subsistencia ni nuestra principal ocupación. 


\section{Conclusiones}

Les mbya crean diversos proyectos para recrearse como pueblo en los que integran prácticas de la sociedad jurua para distinguirse de ella. Una de estas prácticas es el recurso al código de la "cultura" en las interlocuciones. Estos proyectos se delinean en coyunturas específicas y ante diversas trayectorias en el marco de la historia de las relaciones con colectivos no indígenas y diversas instituciones del Estado. La política de la autonomía mbyaen los casos analizados se establece así de diversas maneras: a través de la incorporación particular de instituciones y prácticas no indígenas, como en el caso de la lucha por la gestión de la escuela o el trabajo junto con el colectivo audiovisual, al mostrar prácticas "culturales" distintivas (versiones de la "cultura" ante otres), como en el caso de los espectáculos y grabaciones corales, y al manejar la preservación de información sobre sus prácticas y los sentidos de estas, como en el caso de la relación de dialogo progresivo con les maestres jurua.

En el ámbito escolar, para muches mbya el ingreso al sistema educativo contribuye a un proyecto de autonomía, en tanto otorga herramientas para posicionarse en diversos espacios del mundo no mbya. Por su parte, les docentes jurua apoyan este proyecto, pero, desde su posición de mayor integración al Estado, se encuentran ante dilemas de interpretación y prejuicios sobre lo que les parece diferente. Sin embargo en el cotidiano tienen la opción de aprender que, como apunta Bartomeu Meliá la acción pedagógica para la alteridad no es un aporte de la sociedad nacional a les indígenas, sino una muestra de lo que estos pueden ofrecer a la sociedad nacional (Meliá, 1999). Vale señalar también que, aunque existen interacciones con jurua para discutir educación y "cultura" mbya, no existen espacios instituidos donde les mbya discutan "cultura" y educación jurua con estos últimos (salvando la excepción de les niñes que cursan EIB). Si bien esta instancia puede no ser necesaria para les mbya, entiendo que puede ser un importante espacio de formación para les jurua que trabajamos con elles, tanto docentes como otres colaboradores, útil para profundizar nuestros aprendizajes autoreflexivos.

En el caso del colectivo de trabajo audiovisual, nuestra colaboración buscó contribuir con la autonomía mbya, realizando consultas ante cada emprendimiento. Esta posición nos otorgó una importante aceptación entre les participantes mbya. No obstante, precisamente el hecho de no tener una convivencia cotidiana extendida en el tiempo nos traía menos desafíos para tal aceptación que los que asumen les docentes en el día a día. Asimismo el hecho de no problematizar enfáticamente nuestras posiciones como científiques sociales, también restringió nuestras interacciones y, tal vez, la posibilidad de generar acciones conjuntas más sólidas y a largo plazo junto con la comunidad e incluso junto con les docentes jurua. Sin embargo, para todes les integrantes, la experiencia movilizó reflexiones sobre nuestros modos de existencia y posiciones sociales.

Entendiendo las posibilidades de la antropología para transformar nuestros modos de conocer y nuestra capacidad de intervenir en el status quo, este análisis ha buscado contribuir al respeto de las búsquedas de singularidad y de autonomía mbya y a pensar modos de buscar mayor fluidez y reflexividad jurua en los diálogos interétnicos cotidianos.

\section{Agradecimientos}

Las reflexiones aquí presentadas que parten de una investigación que aborda procesos 
de trabajo centrados en la cultura entre les qom del oeste de Formosa y les mbya de Misiones realizada en parte con una beca pos doctoral del CONICET (Greco 2016). Agradezco al colectivo Tataypy Rupa, a Marilyn Cebolla, Ana Padawer, Macarena Ossola y Eugenia Flores por sus contribuciones y reflexiones, así como a las comunidades mbya de San Ignacio que abrieron sus puertas. 


\section{Q Referencias Bibliográficas}

》Abu Lughod, L. (2012). Escribir contra la cultura. Andamios, 9(19), 129-157.

»Albert, B. (1997). Situation ethnographique et mouvements ethniques: notes sur le terrain post-malinowskien. En M. Agier (Org.), Anthropologues en Danger. L'engangement sur le terrain (pp. 75-88). Paris: Jean Michel Place.

» Assis, V. y Garlet, J. (2004). Análise sobre as populações guarani contemporâneas: demografia, espacialidade e questões fundiárias. Revista de Indias, 64(230), 35-54.

» Bañay, A. (2015). Educación intercultural bilingüe, el desafío en una comunidad mbyaguaraní. La rivada, 3(4), 1-16.

» Barbosa da Silva, A, y Mura, F. (2018). Territory and Domestic Ecology among de Kaiowa of Mato Grosso do Sul. Vibrant, 15(2), 1-24.

» Bartolomé, L. (2007). Los colonos de Apóstoles: estrategias adaptativas y etnicidad en una colonia eslava en Misiones. Posadas: Editorial Universitaria UNAM.

» Bartolomé, M. A. (2009). Parientes de la selva. Los guaraníes mbyà de la Argentina. Asunción: Centro de Estudios Antropológicos de la Universidad Católica.

» Briones, C. (1998). (Meta)cultura del Estado-nación y estado de la (meta)cultura. Serie Antropología, 244, 1-55.

»Briones, C. (2005). Formaciones de alteridad: contextos globales, procesos nacionales y provinciales. En C. Briones (Comp.), Cartografías Argentinas. Políticas Indigenistas y Formaciones Provinciales de Alteridad (pp. 9-40). Buenos Aires: Antropofagia.

"Cabral de Oliveira, J. y Keese dos Santos, L. (2014). "Perguntas demais": multiplicidade de modos de conhecer em uma experiência de formação de pesquisadores Guaraní Mbya. En M. Carneiro da Cunha y P. Niemeyer Cesarino (Orgs.), Políticas Culturais e Povos Indígenas (pp. 113-134). São Paulo: Cultura Acadêmica Editora.

» Cadogan, L. (1960). En torno de la aculturación de los Mbyá Guaraní del Guairá. América Indígena 20(2), 133-150.

»Cantore, A. y Boffelli, C. (2017). Etnicidad mbya en Puerto Iguazú: Explotación turística de/en comunidades indígenas en la triple frontera (Misiones, Argentina). Runa, 38(2), 53-69.

» Cardoso de Oliveira, R. (2003). Identidade étnica, identificação e manipulação. Sociedade e Cultura, 6(2), 117-131.

»Carneiro da Cunha, M. (2009). Cultura com Aspas e Outros Ensaios. São Paulo: Cosac Naify.

»Cebolla Badie, M. (2005). Docentes y niños: jurua kuery e indios. Breve reseña sobre la situación de las escuelas aborígenes bilingües biculturales en la provincia de Misiones, Argentina. AIBR. Revista de Antropología Iberoamericana, 41, 1-14.

»Cebolla Badie, M. (2017). Cosmología y Naturaleza MbyaGuaraní. Buenos Aires: Editorial Biblos.

»Cebolla Badie, M. y Gallero, C. (2012). Las relaciones blanco-indio a través del registro fotográfico en Misiones, Argentina (1920-1960). En M. Giordano y A. Reyero (Eds.), Identidades en Foco. Fotografía e Investigación Social (pp. 91-110). Resistencia: Instituto de 
Investigaciones Geohistóricas (IIGHI - CONICET) - UNNE.

»Chase-Sardi, M. (1989). El Tekoha. Su organización social y los efectos negativos de la deforestación entre los mbyá-guaraní. Suplemento Antropológico, 24(2), 33-41.

»Clastres, H. (1993). La Tierra Sin Mal. Buenos Aires: Ediciones del Sol.

» Cretton, V. (2010). Mbya reko e elementos urbanos: encontros dos Guarani Mbya com a cidade em contextos distintos de ocupação no Estado do Rio de Janeiro. Intratextos, $1,85-102$.

»Deleuze, G. y Guattari, F. (1995). Mil Platôs. Capitalismo e Esquizofrenia, 2. Sao Paulo: Editora 34.

» Dietz, G. (2017). Interculturalidad: una aproximación antropológica. Perfiles Educativos, 39(156), 192-207.

»Escobar, T. (2015). La Belleza de los Otros. Buenos Aires: Edhasa

»Enriz, N. (2010). Um sonho branco. Reflexões sobre a educação mbya guarani na Argentina. Amazônica, 2(1), 28-44.

»Enriz, N. (2011). Políticas públicas para familias indígenas en Misiones. Runa, 32(1), 27-43.

» Garlet, I. (1997). Mobilidade mbya: História e significação. (Tesis de maestria). Pontifícia Universidade Católica do Rio Grande do Sul, Curso de Pós graduação em História, Instituto de Filosofia e Ciências Humanas, Porto Alegre, Brasil.

» Golé, C. y Rodríguez Celín, M. L. (2017). Reconfiguraciones del vínculo entre escuelas y la diversidad étnica: criollos, mbyà-guaraníes y colonos en el sudoeste misionero desde principios del siglo XX. Anuario Historia de la Educación, SAHE, 18(2), 228-250.

» Gómez, M., Greco, L., Torres Agüero S. (2013). Bailar como los antiguos: silencios, memoria y corporalidad entre jóvenes, mujeres y maestros bilingües del Barrio Toba de Ingeniero Juárez. En Actas del X Reunión de Antropología del MERCOSUR (RAM). Córdoba: Universidad Nacional de Córdoba.

» Gordillo, G. (1996). Entre el monte y las cosechas: Migraciones estacionales y retención de fuerza de trabajo entre los tobas del oeste de Formosa (Argentina). Estudios Migratorios Latinoamericanos, 11(32), 135-167.

» Gorosito Kramer, A. (1982). Encuentros y Desencuentros. Relaciones Interétnicas y Representaciones en Misiones, Argentina. (Tesis de Maestría). Universidade de Brasília, Programa de Pós Graduação em Antropologia Social, Brasilia, Brasil.

» Gorosito Kramer, A. (2006). Liderazgos guaraníes: breve revisión histórica y nuevas notas sobre la cuestión. Avá, 9, 11-27.

» Greco, L. (2016). “Mi cultura la tengo, pero no la practico”. Reflexiones sobre las movilizaciones de la cultura y los pueblos indígenas en Ingeniero Juárez, Formosa, Argentina. Etnografías Contemporáneas, 2(3), 176-203.

" Lins Ribeiro, G. (2008). Diversidade cultural enquanto discurso global. En Actas del IX Congreso Argentino de Antropología social. Posadas: Universidad Nacional de Misones (UNAM).

» Macedo, V. (2013). De Encontros nos Corpos Guarani. ILHA, 15(2), 181-210.

» Meliá, B. (1981). El “modo de ser” guaraní en la primera documentación jesuítica (15941639). Revista de Antropologia, 24, 1-24.

» Meliá, B. (1986). El guaraní conquistado y reducido. Ensayos de etnohistoria. Asunción. Centro de Estudios Antropológicos, Universidad Católica de Asunción.

» Meliá, B. (1999). Educação indígena na escola. Cadernos Cedes, 19(49), 11-17. 
»Padawer, A., Canciani, L., Hlebovich M., Kostlin, L. y Vidal M. (2013). Situación Socioeducativa en las Colonias Aparicio Cue-Pastoreo-Invernada (San Ignacio- Misiones). Informe para el Proyecto de Voluntariado Universitario dirigido por el Mg. A. Oviedo. Universidad Nacional de Misiones.

»Pissolato, E. (curadora). (2012.) Tapé Porã, Impressões e Movimento Mbya. O Guarani Mbyano Rio de Janeiro. Catálogo de muestra. FUNAI. Rio de Janeiro. Recuperado de http://issuu.com/priscillamoura/docs/catalogo_mi_10_dez-final-oo2_em_ba.

»Rappaport, J. (2007). Más allá de la escritura: la epistemología de la etnografía en colaboración. Revista Colombiana de Antropología, 43, 197-229.

»Sahlins, M. (1997). O “pessimismo sentimental” e a experiência etnográfica: por que a cultura não é um “objeto” em via de extinção. MANA, 3(2), 103-150

»Segato, R. (2007). La nación y sus otros. Buenos Aires: Prometeo libros.

»Wagner, R. (2010). A invenção da cultura. São Paulo: Cosacnaify.

»Wilde, G. (2001). Los guaraníes después de la expulsión de los jesuitas: dinámicas políticas y transacciones simbólicas. Revista Complutense de Historia de América, 27: 69-106

»Wilde, G. (2008). Imaginarios contrapuestos de la selva misionera. Una exploración por el relato oficial y las representaciones indígenas sobre el ambiente. En Gestión ambiental y conflicto social en América Latina (pp. 193-225). Buenos Aires: CLACSO.

»Wright, S. (1998). The Politicization of “Culture”. Anthropology Today, 14(1), 7-15.

»Yúdice, G. (2002). El Recurso a la Cultura. Usos de la Cultura en la Era Global. Barcelona. Gedisa. 
